\title{
Tracking transient conformational states of T4 lysozyme at room temperature combining X-ray crystallography and site-directed spin labeling
}

Philipp Consentius ${ }^{1}$, Ulrich Gohlke ${ }^{2}$, Bernhard Loll ${ }^{3}$, Claudia Alings ${ }^{3}$, Robert Müller ${ }^{4}$, Udo Heinemann ${ }^{2,3}$, Martin Kaupp ${ }^{4}$, Markus Wahl ${ }^{3}$, Thomas Risse ${ }^{1,5, *}$

${ }^{1}$ Institute of Chemistry and Biochemistry, Freie Universität Berlin, Takustr. 3, 14195 Berlin, Germany

${ }^{2}$ Max Delbrück Center for Molecular Medicine in the Helmholtz Association, Robert-Rössle-Str. 10, 13125 Berlin, Germany

${ }^{3}$ Institute of Chemistry and Biochemistry, Freie Universität Berlin, Takustr. 6, 14195 Berlin, Germany

${ }^{4}$ Institute of Chemistry, Sekr. C7, Technische Universität Berlin, Straße des 17. Juni 135, 10623 Berlin, Germany

${ }^{5}$ Berlin Joint EPR Laboratory Freie Universität Berlin 


\section{Supplementary Information}

Table S1. Crystallographic data collection and model refinement statistics for the Lysozyme L118C-R1 variant.

\begin{tabular}{|c|c|c|}
\hline \multicolumn{2}{|l|}{ Data Collection } & \multirow[b]{2}{*}{ 5JDT } \\
\hline PDB ID & $5 G 27$ & \\
\hline Wavelength $[\AA ̊]$ & 1.5418 & 0.895 \\
\hline Temperature [K] & 293.15 & 100.00 \\
\hline Space group & $\mathrm{P} 3221$ & $\mathrm{P} 3221$ \\
\hline $\begin{array}{l}\text { Unit Cell Parameters } \\
a=b, c[\AA]\end{array}$ & $60.93,97.00$ & $60.28,96.97$ \\
\hline Resolution range $[\AA]^{a}$ & $46.35-1.61$ (1.64 -1.61) & $20.00-1.00(1.06-1.00)$ \\
\hline \multicolumn{3}{|l|}{ Reflections $^{a}$} \\
\hline Unique & $27,618(1,291)$ & \\
\hline Completeness [\%] & 99.7 (95.9) & 99.9 (100.0) \\
\hline Multiplicity & $5.7(5.0)$ & $7.6(4.8)$ \\
\hline \multicolumn{3}{|l|}{ Data quality ${ }^{\mathrm{a}}$} \\
\hline 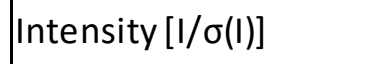 & $13.8(2.4)$ & $14.8(4.1)$ \\
\hline $\mathrm{R}_{\text {meas }}[\%]^{\mathrm{b}}$ & $8.3(82.9)$ & 9.9 (42.9) \\
\hline $\mathrm{CC}_{1 / 2}$ & $0.998(0.520)$ & $0.999(0.895)$ \\
\hline Wilson $B$ value $\left[\AA^{2}\right]$ & 21.74 & 11.50 \\
\hline \multicolumn{3}{|l|}{ Refinement } \\
\hline Resolution range $[\AA]^{a}$ & $19.58-1.61(1.67-1.61)$ & $20.00-1.00(1.04-1.00)$ \\
\hline \multicolumn{3}{|l|}{ Reflections $^{\text {a }}$} \\
\hline Number & $27,529(2,808)$ & $110,399(10,932)$ \\
\hline Completeness [\%] & 99.7 (99.7) & 99.9 (100.0) \\
\hline Test Set (5\%) & $1,340(152)$ & $5,490(546)$ \\
\hline $\mathbf{R}_{\text {work }}{ }^{\mathrm{a}, \mathrm{c}}$ & $0.1505(0.2394)$ & $0.1145(0.1661)$ \\
\hline$R_{\text {free }} e^{a, d}$ & $0.1691(0.2509)$ & 0.1297 (0.1809) \\
\hline
\end{tabular}




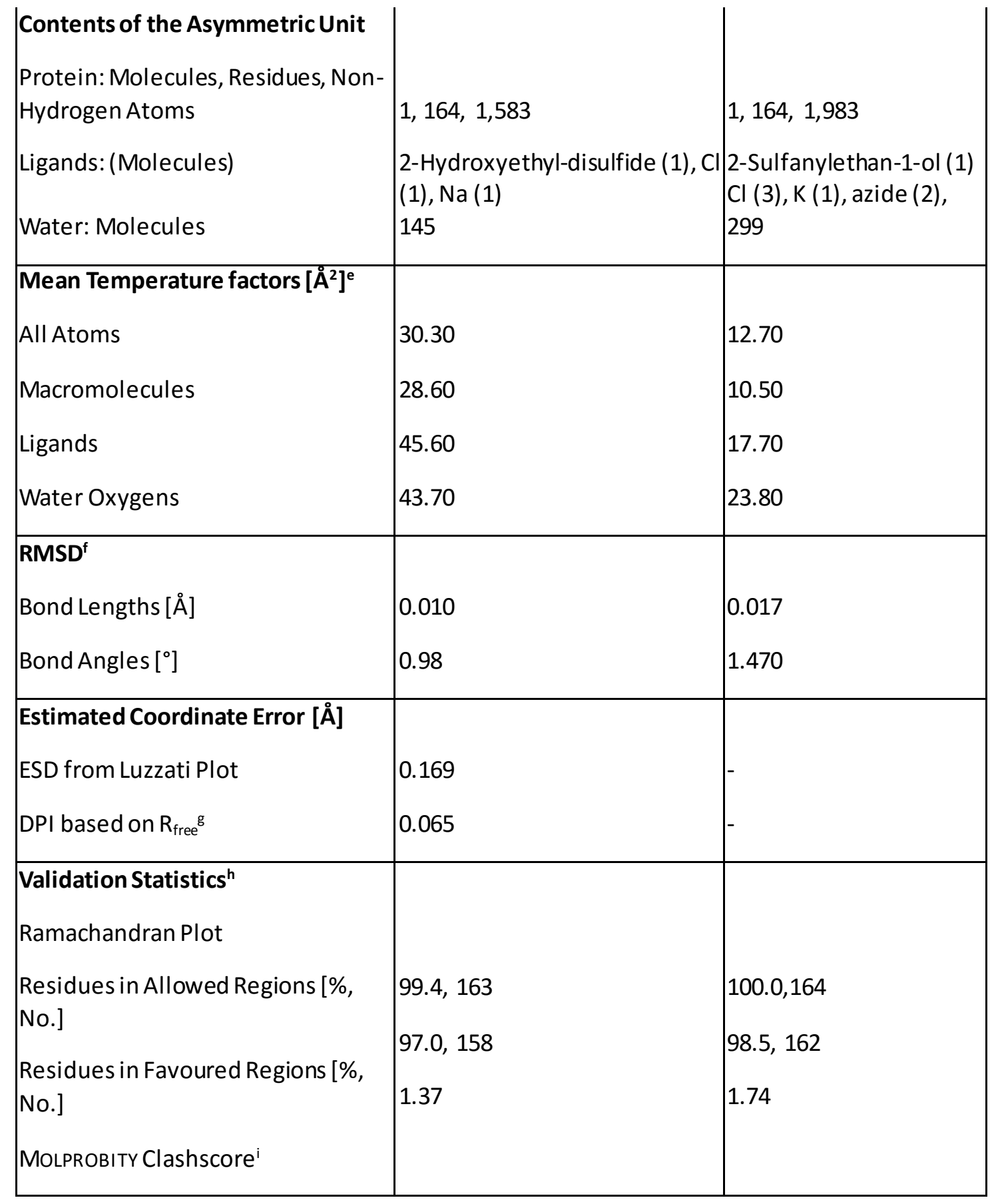

a data for the highest resolution shell in parenthesis

b $\quad \mathrm{R}_{\text {meas }}=\sum_{h}[n / n-1]^{1 / 2} \sum_{i}\left|I_{h}-I_{h, i}\right| / \sum_{h} \sum_{i} I_{h, i}$, where $I_{h}$ is the mean intensity of symmetryequivalent reflections and $n$ is the redundancy

c $\quad \mathrm{R}_{\text {work }}=\sum_{h}\left|F_{o}-F_{c}\right| / \sum F_{o}$ (working set, no $\sigma$ cut-off applied)

d $\quad R_{\text {free }}$ is the same as $R_{\text {work, }}$, but calculated on $5 \%$ of the data excluded from refinement

e calculated with PHENIX ${ }^{[1]}$

$f \quad$ Root-mean-square deviation from target geometries

g definedin ${ }^{[2]}$ 
h calculated with MOLPROBITY[3]

i Clashscore is the number of serious steric overlaps (>0.4) per 1,000 atoms.

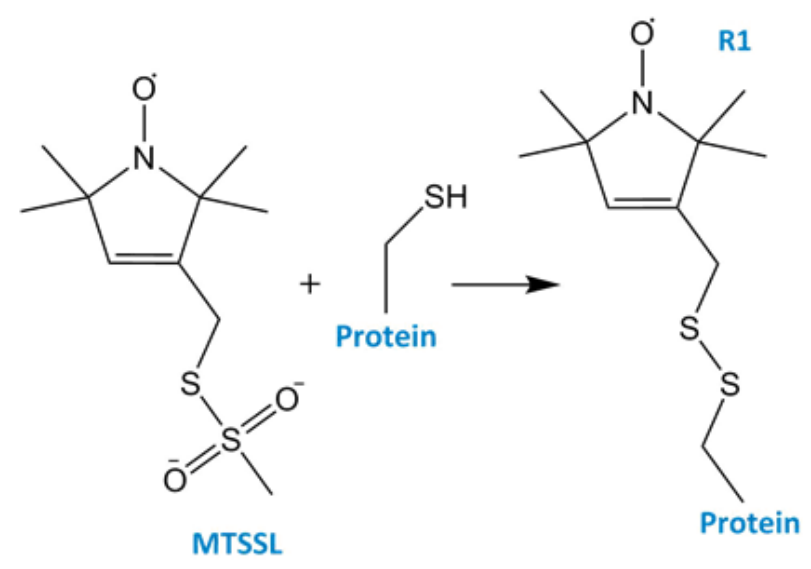

Figure S1. Site-directed spin labeling of a cysteine residue with the spin label 1-Oxyl-2,2,5,5-tetramethyl- $\Delta 3$ pyrroline-3-methyl (Methanethiosulfonate, MTSSL) resulting in a covalent attachment via a disulfide bond. 
A $\quad 1.0 \AA$ A resolution (PDB entry: 5JDT)

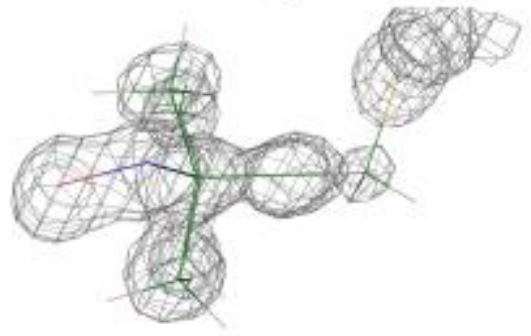

B

1.3 A resolution (unpublished)

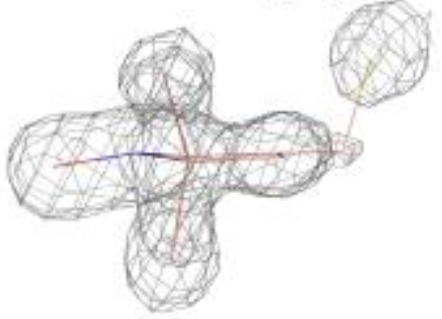

C $\quad 1.8 \AA$ resolution (PDB entry: $2 \mathrm{NTH}$ )

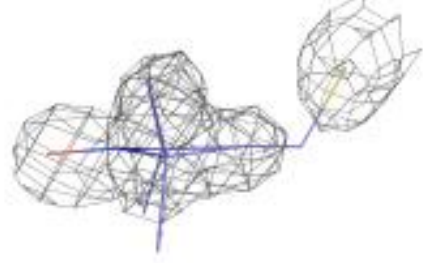

Figure S2. Structural model of the L118R1 side chain refined with a resolution of $1.0 \AA$ (upper panel, green) and 1.3 $\AA$ (middle panel, magenta) superimposed by a $2 \mathrm{~F}_{0}-\mathrm{F}_{\mathrm{c}}$ density difference map $(\sigma=1.3)$ in gray. The structural model of the L118R1 side chain (bottom panel, blue) was visualized from PDB entry 2NTH. The superimposed electron density difference map in gray was taken from the Electron Density Server (EDS) at Uppsala University. 


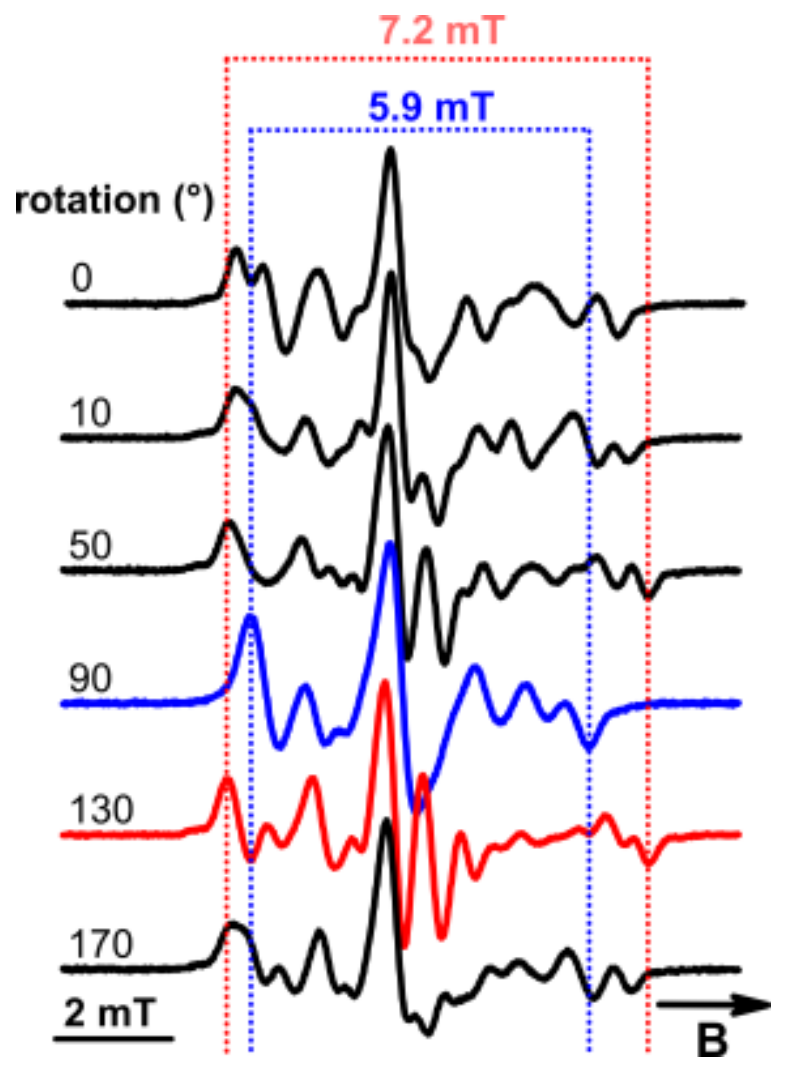

Figure S3. Angle-dependent EPR spectra of a T4L 118R1 singlecrystal. Six characteristic EPR spectra with a rotation of the capillary by $0^{\circ}, 10^{\circ}, 50^{\circ}, 90^{\circ}, 130^{\circ}$ and $170^{\circ}$ are shown. The scan width is $12 \mathrm{mT}$. 


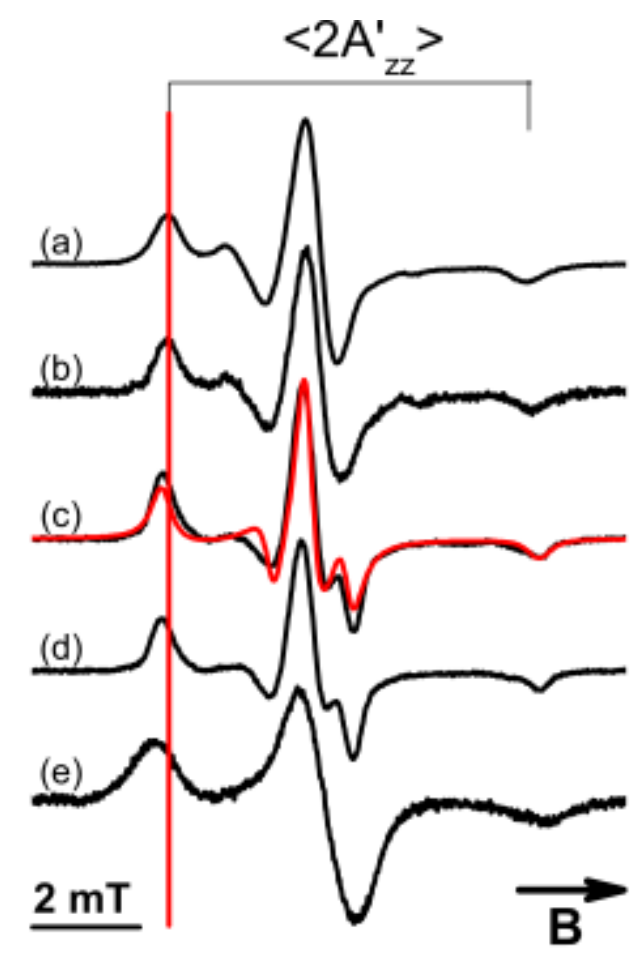

Figure S4. EPR spectra of T4L 118R1 in label buffer (a) and mother liquor (b) recorded with 30\% (w/v) sucrose. A suspension of micro crystals of T4L $118 \mathrm{R} 1$ measured twice (c), (d) with the B field rotated by $90^{\circ}$. A simulation of the line-shape (slow motion model developed by Freed and co-workers) using parameters from Table S2 (red) is superimposed to (c). Spectra a-d were recorded at $298 \mathrm{~K}$ whereas (e) shows the EPR spectrum of crushed crystals measured at $50 \mathrm{~K}$. Vertical line is added as a guide to the eye.

It is noted that the spectrum of the crushed crystals reported here is significantly different from the one presented by Guo et al. ${ }^{[4]}$. In particular, it lacks the rather strong mobile component reported by Guo et al.. Currently we cannot explain this discrepancy. However, the line shape reported here is consistent with other observations such as the line shape of this variant in viscous solution (Figure S4), and we conclude that the spectrum presented here reflects the line shape of an isotropic ensemble of single crystals.

Table S2. Static and dynamic parameters for EPR line-shape simulation

\begin{tabular}{|c|c|c|c|c|c|c|c|c|c|c|c|}
\hline $\mathrm{A}_{\mathrm{x} x}$ & $\mathrm{~A}_{\mathrm{yy}}$ & $\mathrm{A}_{z z}$ & $\mathrm{~g}_{x x}$ & $\mathrm{~g}_{\mathrm{yy}}$ & $\mathrm{g}_{z z}$ & $\log \mathrm{R}_{\mathrm{xx}}$ & $\log \mathrm{R}_{\mathrm{yy}}$ & $\log \mathrm{R}_{z z}$ & $\beta_{\mathrm{D}}$ & $\mathrm{S}$ & $\mathrm{Iw}$ \\
\hline $\begin{array}{c}0.62 \\
\mathrm{mT}\end{array}$ & $\begin{array}{c}0.59 \\
\mathrm{mT}\end{array}$ & $3.7 \mathrm{mT}$ & 2.0086 & 2.0068 & 2.0023 & $\begin{array}{c}7.8- \\
8.2\end{array}$ & $\begin{array}{c}7.8- \\
8.2\end{array}$ & $\begin{array}{c}7.8- \\
8.2\end{array}$ & 0 & 0.91 & $\begin{array}{c}0.17 \\
\mathrm{mT}\end{array}$ \\
\hline
\end{tabular}



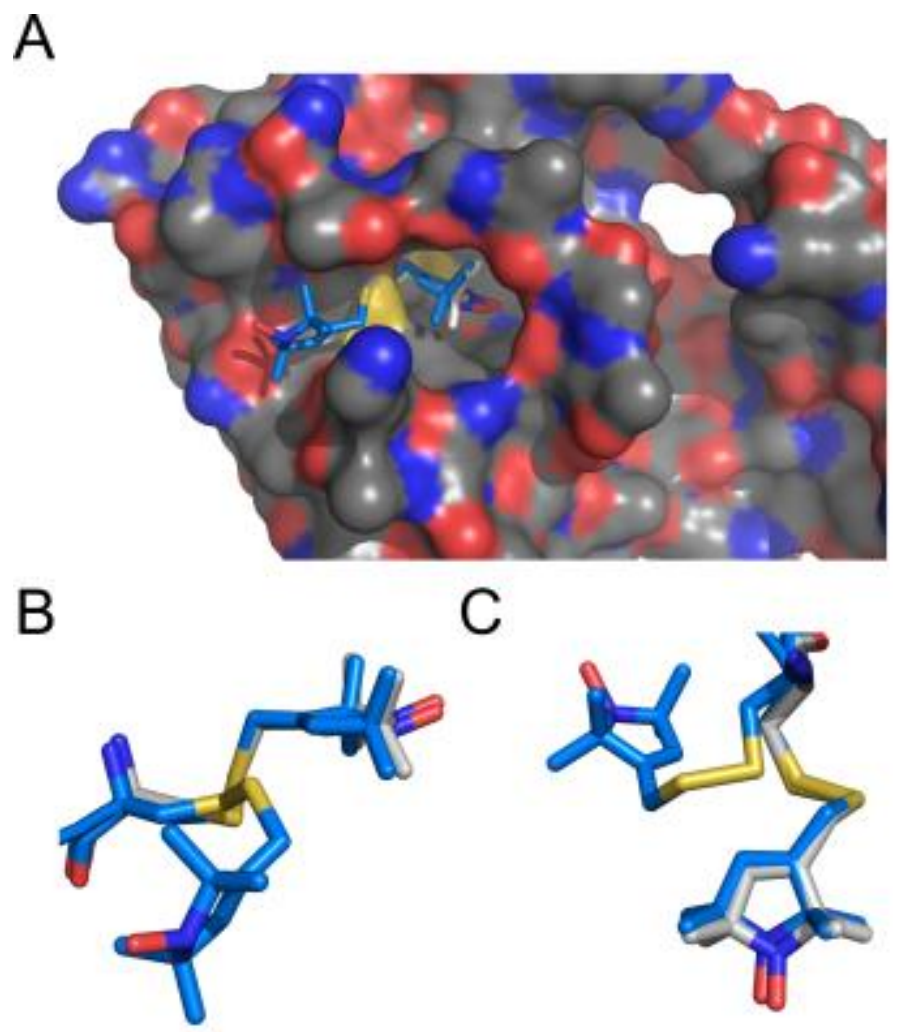

C

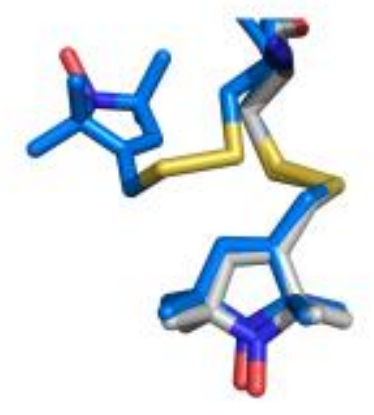

Figure S5. A) Connolly surface plot of the C-terminal domain of T4 Iysozyme representing the single spin label conformation determined at $100 \mathrm{~K}$ in white (LT-conformation) and the two conformations present at room temperature in blue (RT-conformation) in stick representation. (B,C) Stick model of two spin label conformations as determined by X-ray crystallography at room temperature (blue) as well as the one conformation observed at $100 \mathrm{~K}$ (gray): (B) side-view and (C) the top-view on the nitroxidering of the " $\mathrm{LT}$ "-conformation. 
A

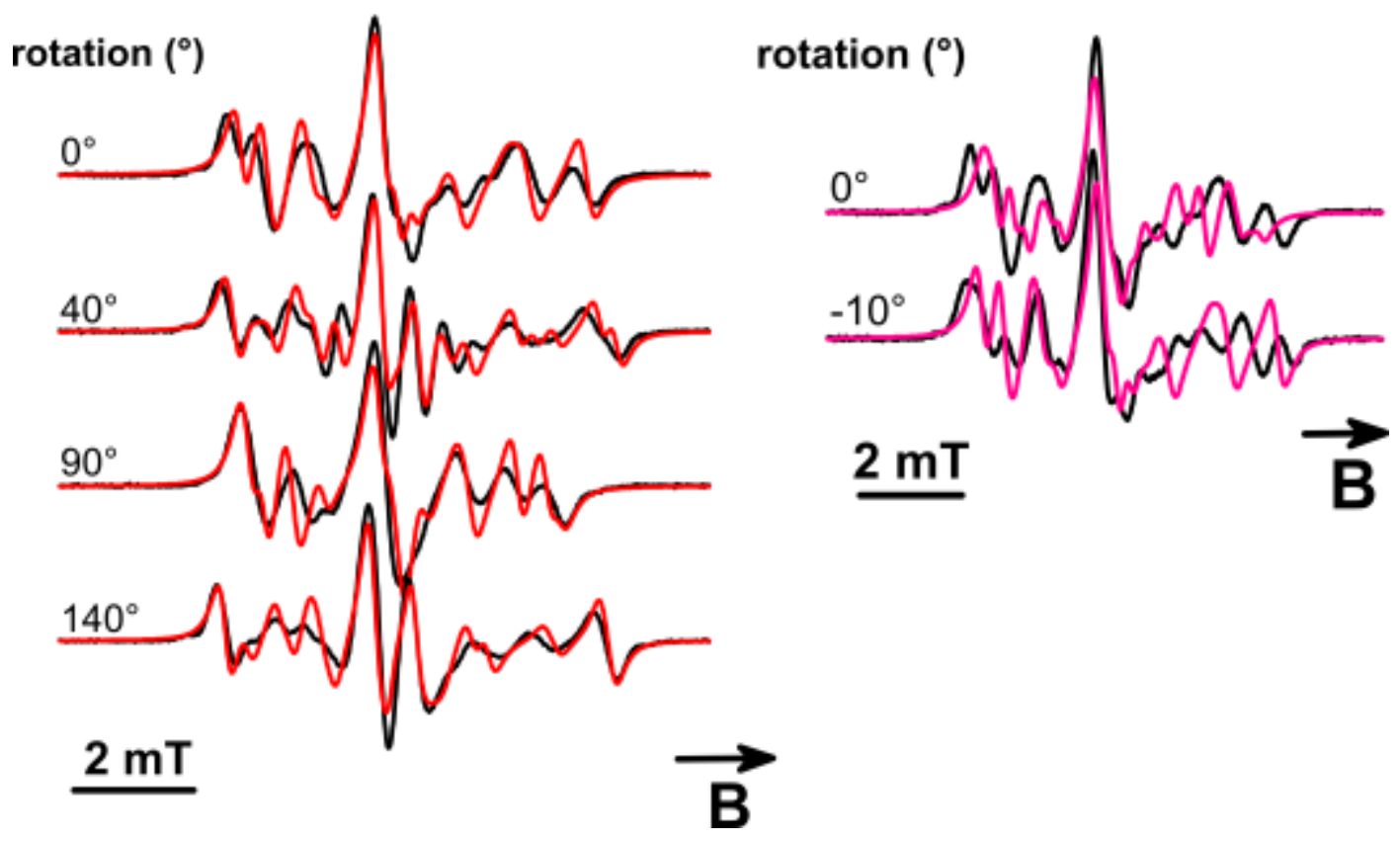

Figure S6. (A) Four spectra out of an angle dependent series taken for a single crystal of a $118 \mathrm{R} 1$ variant of T4 Iysozyme (black traces). Note, that the spectra in Figure 2 and the ones shown here were taken for a different crystal, hence probing a different plane within the unit cell. Angles are given with respect to a reference orientation $\left(0^{\circ}\right)$, for which the orientation of the unit cell was determined by X-ray crystallography. Red traces are simulations to the measured line shape using exactly the same model parameters (Table S2) as orientation of the spin label within the unit cell (order potential) as used to fit the spectrum shown in Figure 2. (B) Top: EPR spectrum of the reference orientation $\left(0^{\circ}\right.$, black) superimposed by a simulation assuming a g- and A-tensor orientation which is rotated by $10^{\circ}$ around the molecular y-axis as compared to the simulation shown in A (magenta). Bottom: EPR spectrum taken at an angle of $-10^{\circ}$ (black) superimposed by the line shape simulation used in $A$ to simulate the spectrum taken at $0^{\circ}$ (magenta). 


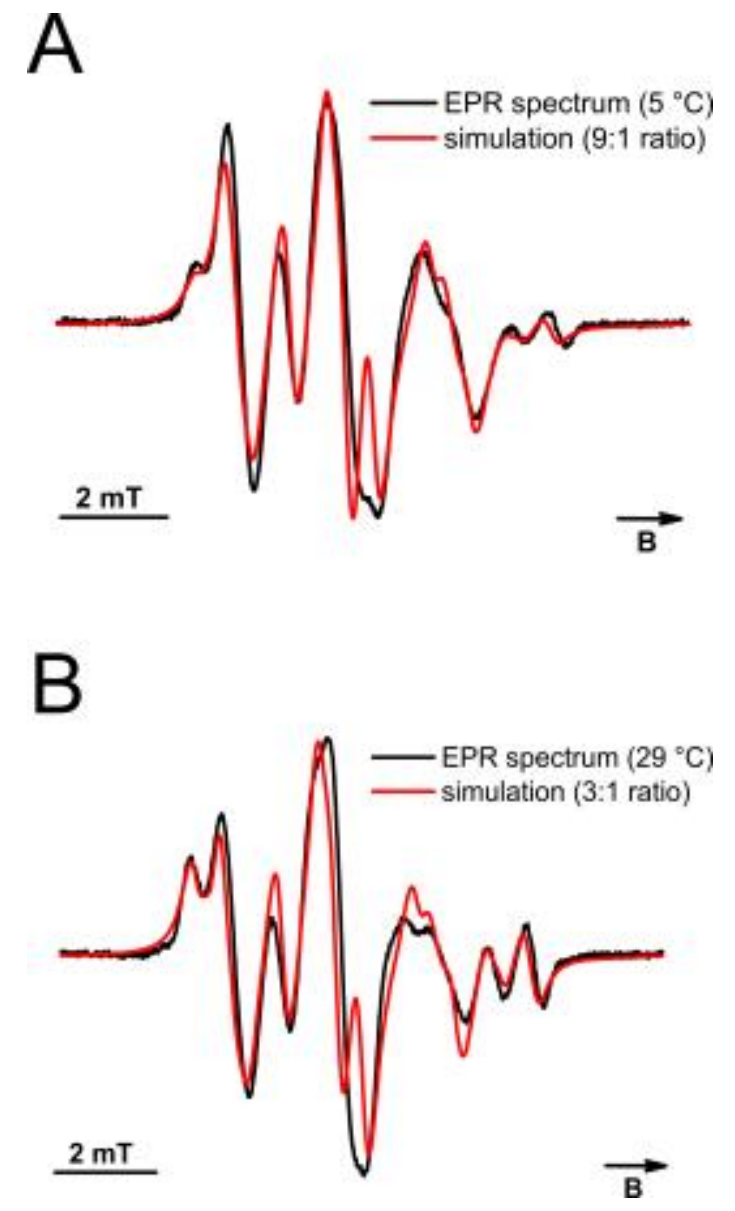

Figure S7. The simulations (red traces) are a superposition of the line shapes for the LT and RT conformation shown in Figure 2 using appropriate relative spectral weight to fit the experimental spectra (black traces) best. (A) spectra taken at $5^{\circ} \mathrm{C}$ simulated by a 9:1 ratio of the LT and the RT conformation. (B) EPR spectrum taken at $29{ }^{\circ} \mathrm{C}$ simulated by a 3:1 ratio of the LT and the RT conformation. 


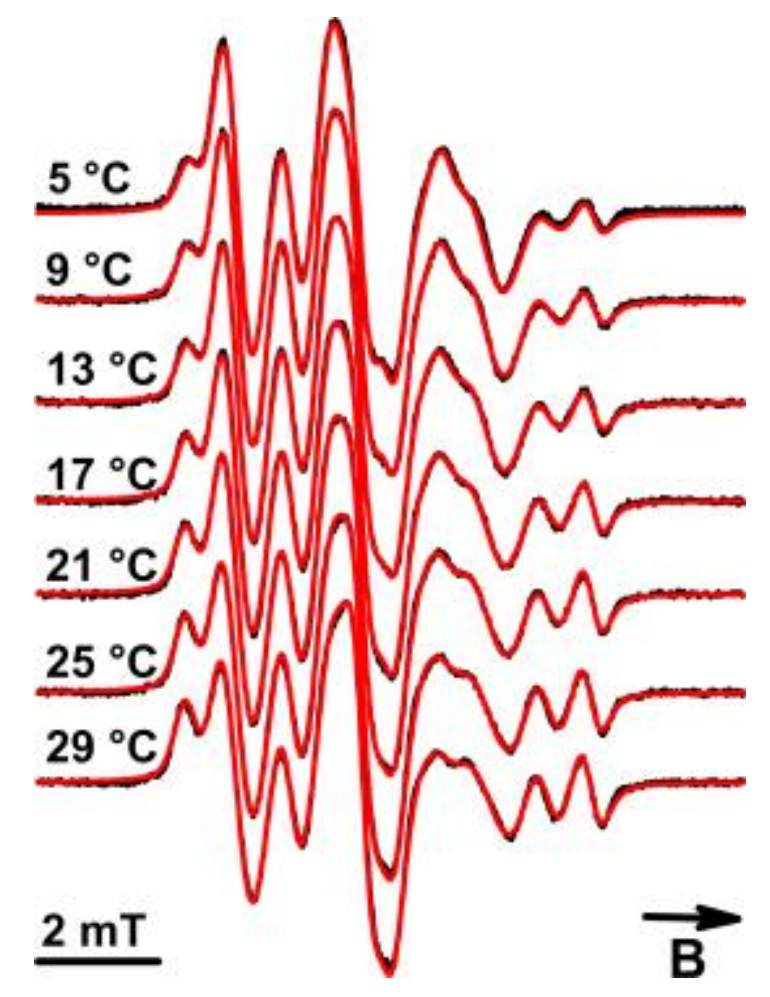

Figure S8. EPR spectra of a T4L L118R1 labeled single crystal (black traces) are superimposed with weighted lineshape fits (red traces) for seven different temperature settings ranging from $5^{\circ} \mathrm{C}$ to $29^{\circ} \mathrm{C}$.

The simulations provided in Figure 2 show that the two lines indicated in Figure $3 \mathrm{~A}$ are exclusively due to absorption from one of the two conformations, respectively. A quantitative analysis of the relative intensities based on these simulations is, however, hampered by the simplified model used to describe the line shape. For a quantitative description, the line shape was modeled using first derivative Voigt lines. One line is used for each of the resonances indicated in Figure 3A. The overall line shape was modeled by describing the rest of the observed line shapes by appropriately chosen derivative Voigt profiles. The choice of these lines was restricted such that all are well separated from the two signals used for the evaluation of the population. The intensities were varied to optimize the fit for each spectrum in the temperature series. In addition, the resonance position and width were adjusted slightly (a few tenth of Gauss) to account for the changes due to the temperature induced modification of the dynamics.

\section{Discussion of the different populations observed in EPR and X-ray crystallography}

The population of the two conformations differs considerably between EPR and X-ray crystallography, and this can be explained by several factors that may affect the determination of the occupancy at a particular atomic position. First, a well-known correlation exists between the occupancy of an atom and its atomic displacement parameter (ADP, also termed B-factor) ${ }^{[5]}$, 
rendering the two indistinguishable at typical resolutions for protein crystals. Therefore, the increase of atomic motion at elevated temperature may lead to a misjudgment of the true occupancy. A possible solution to this problem is to correlate the obtained ADP values for a given set of atoms, forming a side chain for example, with those of atoms in spatial vicinity. In addition, atoms near the surface of a protein with few non-covalent interactions are expected to show larger ADPs than those that are located in the interior. In case of the structure determined in this study at high temperature, the mean ADPs for the buried vs exposed spin label with a ratio of $1: 3$ were ca. $40 \AA^{2}$ and $50 \AA^{2}$, respectively. For the opposite distribution (3:1) for example, the mean ADPs were ca. $57 \AA^{2}$ and $25 \AA^{2}$, which would be in contradiction to their respective exposure to solvent in the crystal lattice. It is important to note that the spin concentration determined by the intensity of the EPR signal prior and after structure determination drops to about $40 \%$ of its original value (Figure S9). The line shape of the spectrum is, however, unchanged indicating that both the conformations and their relative population of the paramagnetic spin labels are retained. It is well known that nitroxide spin label can undergo one electron reduction typically resulting in a diamagnetic hydroxylamine. ${ }^{[6-7]}$ While the reduced form is difficult to discriminate from the paramagnetic nitroxide in diffraction experiments with given resolution, it is expected to have significant impact on the relative stability of the two conformations, as the reduced moiety will lack the ability to form the hydrogen bond stabilizing the low temperature conformation. In turn, the reduced spin labels should exhibit an increased population of the high temperature conformation.

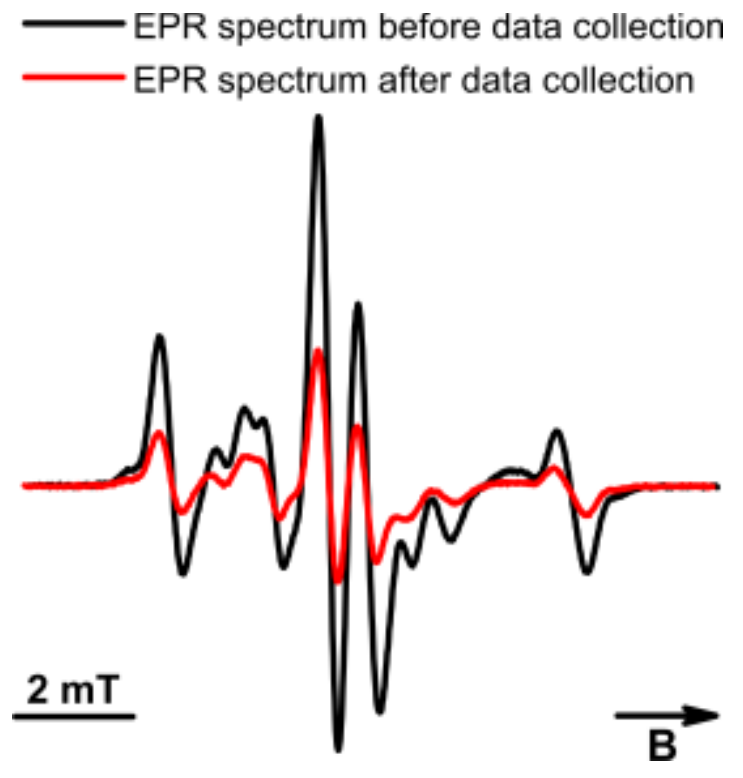

Figure S9. EPR spectra of a T4L L118R1 labeled single crystal obtained before (black trace) and after (red trace) Xray diffraction data collection of a complete data set (approx. 2 days) at room temperature. The total signal intensity was reduced to about $40 \%$ after the data collection experiment. 


\section{Quantum-chemical calculations}

In all calculations a simplified structural model of the nitroxide-containing L118R1 side chain has been employed, in which the sulfur link (see Figures S1 and S7) was replaced by a hydrogen atom. Model 1 exhibits a planar arrangement around the nitrogen atom (with an improper dihedral angle $\theta(\mathrm{NCCO})$ of $\left.0.0^{\circ}\right)$, while model 2 preserves the pyramidal environment with $\theta(\mathrm{NCCO})=17.2^{\circ}$ from the crystal structure data.
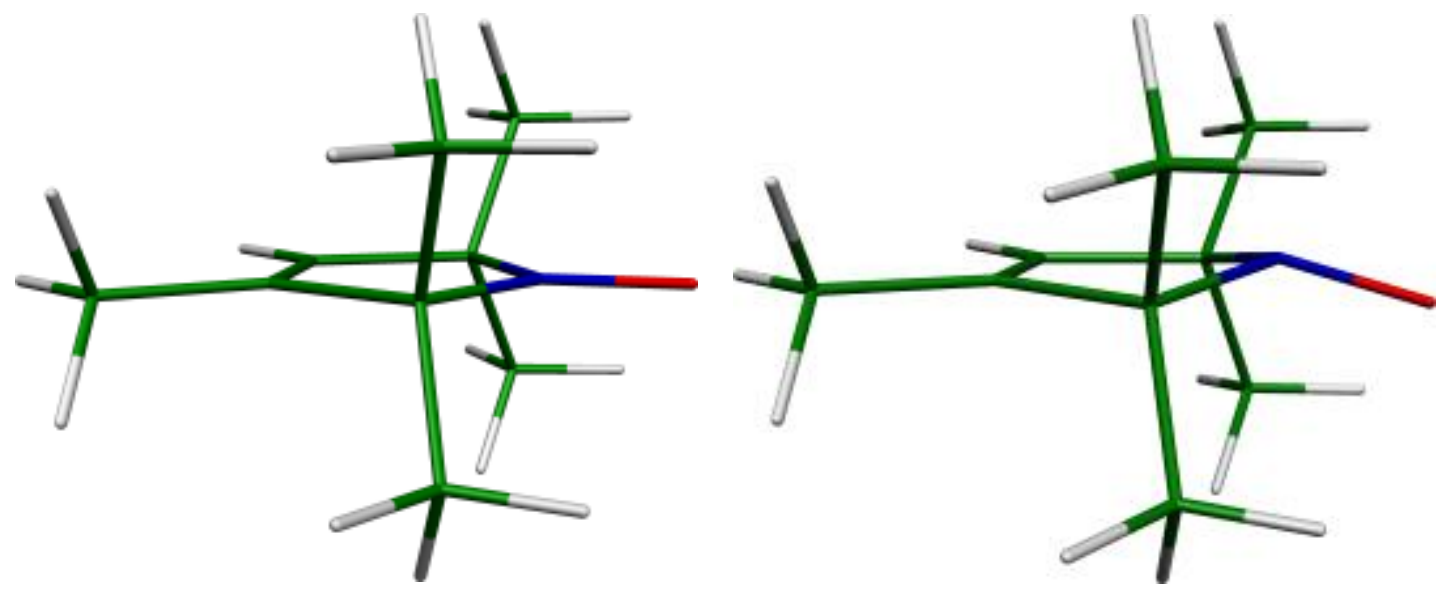

Figure S10. Structural models (left: model 1 with planar arrangement around N; right: model 2 with preserved pyramidalization around N) used for g- and A-tensor calculations (B3LYP-D3(BJ)/def2-TZVPP).

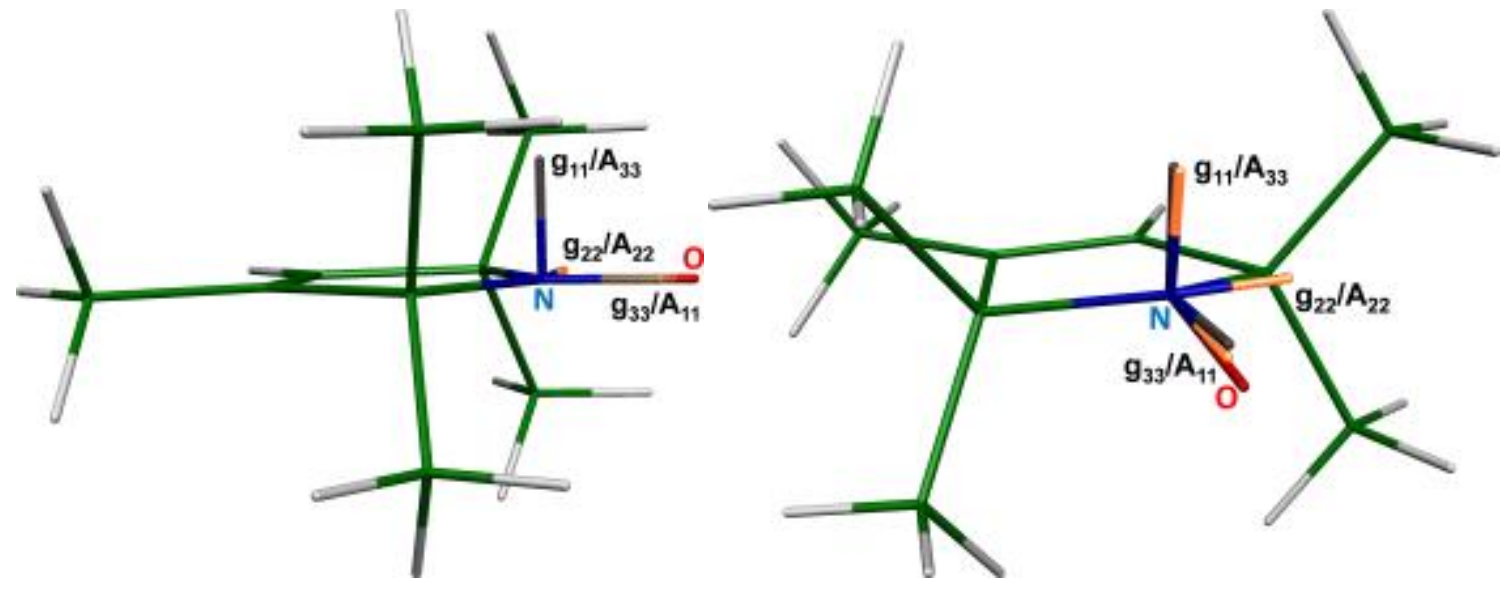

Figure S11. Orientation of the g- and A-tensor principal axes (unit vectors; g-tensor: bright orange coloring; Atensor: grey coloring) in two structural models (left: model 1; right: model 2), from B3LYP-D3(BJ)/IGLO-III calculations. 


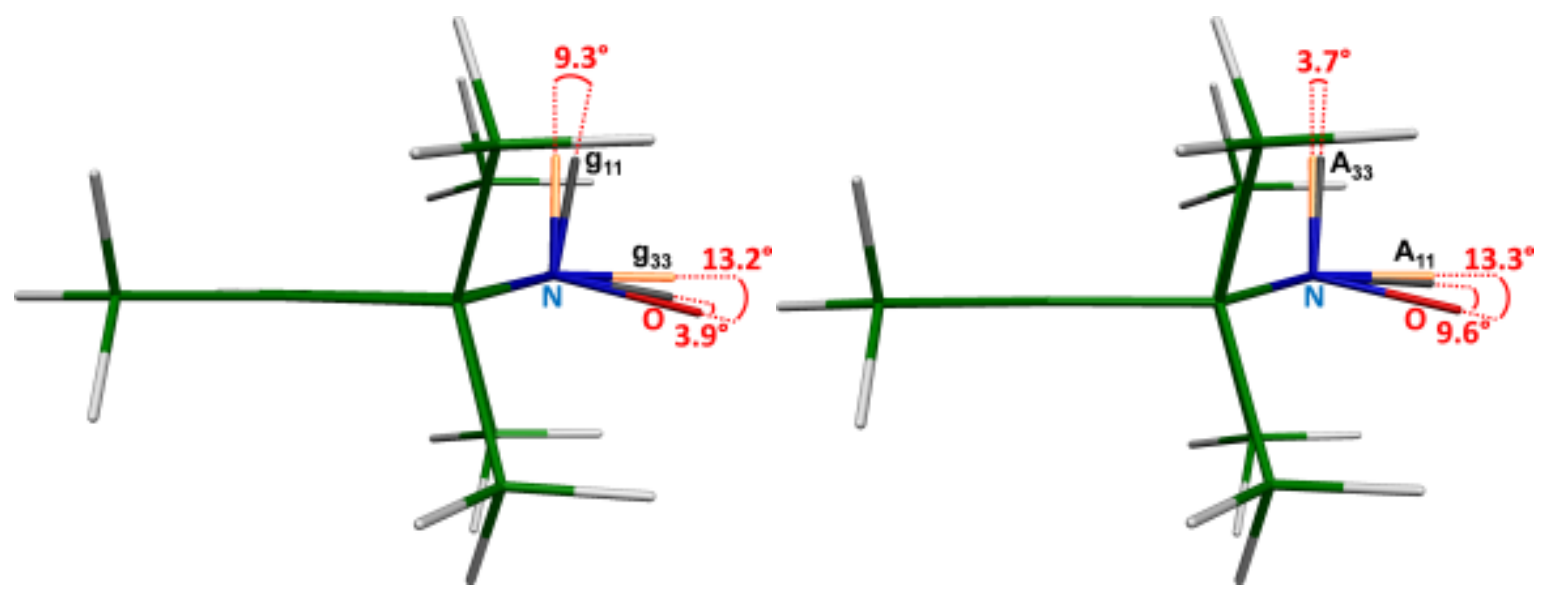

Figure S12. Comparison of the orientation of the g-tensor (left) and A-tensor (right) principal axes (unit vectors) of model 1 (bright orange coloring) and model 2 (grey coloring). The molecular structure of model 1 is omitted in these representations, and the corresponding unit vectors have been centered at the nitrogen atom of model 2 for clarity (B3LYP/IGLO-III//B3LYP-D3(BJ)/def2-TZVPP).

\section{References}

[1] P. D. Adams, P. V. Afonine, G. Bunkoczi, V. B. Chen, I. W. Davis, N. Echols, J. J. Headd, L. W. Hung, G. J. Kapral, R. W. Grosse-Kunstleve, A. J. McCoy, N. W. Moriarty, R. Oeffner, R. J. Read, D. C. Richardson, J. S. Richardson, T. C. Terwilliger, P. H. Zwart, Acta Crystallogr. D 2010, 66, 213-221.

[2] D. W. J. Cruickshank, Acta Crystallogr. D 1999, 55, 583-601.

[3] V. B. Chen, W. B. Arendall, J. J. Headd, D. A. Keedy, R. M. Immormino, G. J. Kapral, L. W. Murray, J. S. Richardson, D. C. Richardson, Acta Crystallogr. D 2010, 66, 12-21.

[4] Z. F. Guo, D. Cascio, K. Hideg, T. Kalai, W. L. Hubbell, Protein Sci. 2007, 16, 1069-1086.

[5] O. Carugo, Protein Eng. 1999, 12, 1021-1024.

[6] R. D. Kornberg, H. M. McConnell, Biochemistry 1971, 10, 1111-\&.

[7] G. Thomas, J. G. Mohanty, Indian J. Chem. A 1982, 21, 451-455. 